\title{
Warp Tension Detection Method of Carbon Fiber Multilayer Loom
}

\author{
Xi Lu ${ }^{1, a}$, Jiancheng Yang ${ }^{1, b}$ \\ ${ }^{1}$ School of Mechanical Engineering, Tianjin Polytechnic University, Tianjin 300387, China; \\ aluxi0425@163.com, byjcg589@163.com
}

Keywords: Multi layer loom, fuzzy control, information fusion, warp tension.

\begin{abstract}
The warp tension, which is the let-off mechanism of carbon fiber multilayer loom, for the study object, the article expounds the composition and distribution of tension sensor, and analysis the detection principle of warp tension, thus design a detection scheme of warp tension. In order to solve the problems of warp tension control accuracy, through the study of neural network, fuzzy control theory and multi sensor information fusion, the authors propose a comprehensive algorithm, which contains neural network, fuzzy control theory and multi sensor information fusion. The weights, which function are obtained by the neural network, and are obtained by the chaos optimization method, then are fused by fuzzy comprehensive evaluation. Experimental results show that the recognition accuracy of multi sensor information fusion better than single sensor, and the accuracy of the tension control of carbon fiber multi-layer fabric loom is improved.
\end{abstract}

\section{Introduction}

Carbon fiber multi-layer loom from Tianjin Polytechnic University of technology to undertake the "12th Five-Year" Plan key projects supported by National Science and Technology "carbon fiber multi-layer Angle weaving equipment and technology". The project has passed by the ministry of science and technology acceptance, for three dimensional fabric weaving has reached domestic advanced level, however, when weaving variable cross-section fabric, there is the long distance, more section and the layer number of warp caused prolapse, and less warp tension testing point, the warp tension control accuracy is lower, and affect the quality of fabric directly. Therefore, warp tension detection accuracy of carbon fiber multi-layer loom and optimization control algorithm become the core problem of the equipment research. Many domestic scholars studied the warp tension test, such as setting up kinematic model of the magnets for hall type tension sensor probe, and putting forward the dynamic tension signal extraction method[1].

To test the tension by the sensor, which are installed on the activity back rest. Many scholars testing tension on the activity back rest[2,3]. However, in carbon fiber multi-layer loom, due to the particularity of carbon fiber, the warp in the process of long distance transmission appear prolapse, it is difficult to detect the warp tension accurately that only install sensors in activity back rest. So we need to install sensors in multiple locations. Multi-sensor distributed data fusion has many practical applications, but rarely applied in the electronic let-off mechanism reported. Loom let off system generally includes control system, driving system, the warp beam, warp, etc., due to the warp by opening, beating-up, send books to take effect, loom warp tension system is time-variant and nonlinear system, it is difficult to establish accurate control model. This paper presents a fuzzy information fusion algorithm of neural network, Fusing the fuzzy theories and the merits of neural network, this method has made up each other's insufficiencies, and has effectively solved questions above.

\section{Tension Detection Principle of Multilayer Warp}

Delivery system including the warp unwinding parts, components parts and yarn tension rope and winding warp parts etc.. As shown in Fig.1, Tension control device including Film tension servo adjusting device, single yarn unwinding device, Yarn tension measuring roller, Five roller tension device[4] . Because of the characteristics of carbon fiber with high shear strength, high specific 
modulus, and self - lubricating property, such a long distance transport of carbon fiber due to self weight and sag, which causes the precision control of warp tension is low. To ensure the constant tension system control of sheet yarn warping, we must be added to the various links of the corresponding sensor to detect tension, which detection of single yarn tension and real-time detection of yarn tension. A displacement sensor and pressure sensor are installed on the tension part of the dual roller type tension compensation mechanism, and the tension sensor is mounted on the tension measuring roller, and the tension sensor is installed on the first and fifth tension rollers in the five roll yarn tension device.

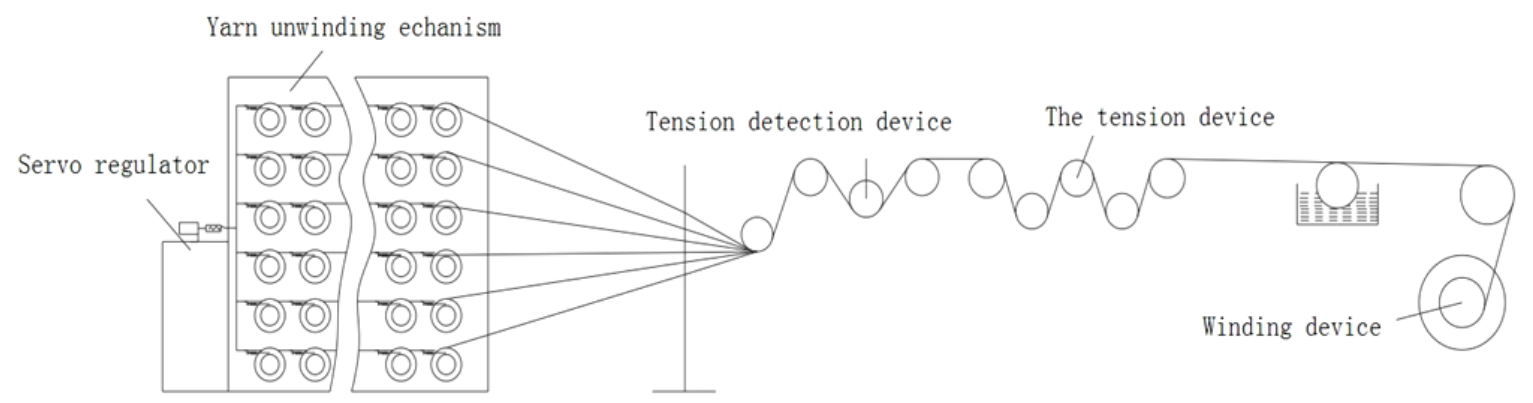

Fig.1 Let-off mechanism of multilayer loom

\section{Fuzzy Fusion Algorithm for Neural Networks}

\subsection{Fuzzy synthetic discrimination.}

Fuzzy comprehensive evaluation includes three elements: (1)Factor set of tension state: $U=\left\{u_{1}, u_{2}, \cdots, u_{n}\right\}$; (2)A sensor detects the tension of the com $m$ ent $V=\left\{v_{1}, v_{2}, \cdots, v_{m}\right\}$. (B)ty relation matrix $R=\left(r_{i j}\right)_{m \times n}$ of $u_{i}(j=1,2, \cdots, n)$ by $v_{i}(i=1,2, \cdots, m)$. In the process of judging, called $a_{i}(i=1,2, \cdots, m)$ factor weight. Fuzzy vector $A=\left(a_{1}, a_{2}, \cdots, a_{m}\right)$ represents the credibility of various sensor judgments.

I selected weighted average $M(\bullet,+)$ as a comprehensive evaluation model $M\left(\hat{*}^{*}{ }^{v}\right)$, that is $p_{j}=\sum_{i=1}^{m}\left(a_{i} \bullet r_{i j}\right)(j=1,2, \cdots, n)$. As the fuzzy vector $\mathrm{R}$ and the fuzzy relational matrix A are known, Fuzzy transformation is used to carry out fuzzy inference.

$$
P=A \circ R=\left(p_{1}, p_{2}, \cdots, p_{n}\right)
$$

Thus, the comprehensive evaluation result set $\mathrm{P}$ mainly depends on the weight set $\mathrm{A}$. so the determination of weights in fuzzy comprehensive evaluation is a key point.

\subsection{A chaotic optimization neural network method for automatically acquiring weights}

Because of the data fusion, between the detection parameters and the multi sensor information fusion data of the subsystems, is the uncertainty, ambiguity and high nonlinearity [5]. Therefore, we combine with the fuzzy theory and neural network theory, through the learning function of neural network to obtain the information of the system parameters and the multi sensor information fusion between the validity of the network weight coefficient [6], then converted into fuzzy comprehensive evaluation of the required weight.The input value of the function-chain neural network is: $u_{1}, u_{2}, \cdots, u_{j}, \cdots, u_{n}$. The output value $y^{\text {est }}$ is:

$$
y^{\text {est }}=\sum_{j=1}^{n} u_{j}(k) \omega_{j}(k)
$$

In the process of training samples, in the global range, the output value of the output of the function chain neural network and the mean variance, which matching the input values of step $\mathrm{k}$, that the minimum value is:

$$
\min \sum_{i=1}^{N}\left[x_{i}^{\text {est }}(k)-x_{i}\right]^{2}=\min \sum_{i=1}^{N}\left[\left(\begin{array}{l}
\omega_{0}(k)+\omega_{1}(k) x_{i} \\
+\omega_{2}(x) x_{i}^{2}+\omega_{3}(k) x_{i}^{3}
\end{array}\right)-x_{i}\right]^{2} \text { s.t. } \quad a_{j} \leq \omega_{j} \leq b_{j}
$$


The minimum value is the function of weight $\omega_{j}(j=1,2, \cdots, n)$.

In the range [0,1] of $\omega_{j}$, which is known. After the optimal solution $\omega_{j}(j=1,2, \cdots, n)$ is obtained by using the variable scale chaos optimization algorithm[7], making the following conversion:

$$
a_{j}=\omega_{j} / \sum_{j=1}^{n} \omega_{j}
$$

\subsection{Determination of fuzzy relational matrix}

Due to the state of the warp tension is affected by many factors, in order to facilitate the research, I choose the five warp tension sensors, respectively, indicating: a sensor 1, a sensor 2, a sensor3, a sensor4, a sensor 5. The decision fusion is adopted in this paper. As shown in figure 2.

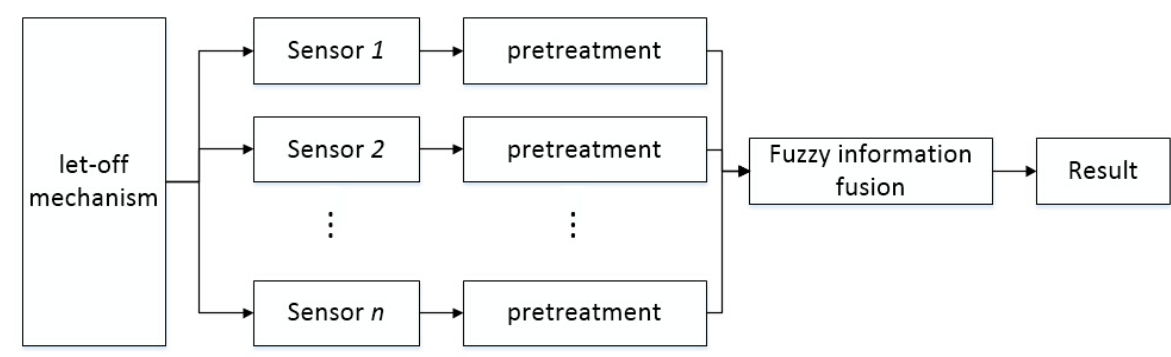

Fig.2 Fusion system of multi sensor fuzzy information fusion system

In this structure, multiple sensors are used to detect the warp tension. For each sensor, the membership value indicate the possibility of a warp tension state, you can get a total of 5 groups of 15 fuzzy membership values. Application of multi-sensor fuzzy fusion theory to 5 groups of membership degree values fusion processing, five kinds of sensor fusion after warp tension state membership degree is obtained, according to certain rules of the decision of the warp tension is judged.

The warp tension is divided into 3 Fuzzy states: small, moderate, large. $H_{0} 、 H_{1} 、 H_{2}$ respectively, With $i, j$ respectively represent different sensors and warp tension state. The membership function is constructed by using Cauchy fuzzy set[8].According to $\mu_{i j}\left(0 \leq \mu_{i j} \leq 1\right)$ can get fuzzy matrix $R^{*}=\left\{\mu_{i j}^{*}\right\}$.

The fuzzy vector can be obtained from the weight of the sensor $A=\left(a_{1}, a_{2}, a_{3}, a_{4}, a_{5}\right)$.According to linear transformation can be

$$
P=A \bullet R^{*}=\left[a_{1}, a_{2}, a_{3}, a_{4}, a_{5}\right] \bullet\left[\begin{array}{ccc}
\mu_{1 H_{0}}^{*} & \mu_{1 H_{1}}^{*} & \mu_{1 H_{2}}^{*} \\
\mu_{2 H_{0}}^{*} & \mu_{2 H_{1}}^{*} & \mu_{2 H_{2}}^{*} \\
\mu_{3 H_{0}}^{*} & \mu_{2 H_{1}}^{*} & \mu_{3 H_{2}}^{*} \\
\mu_{4 H_{0}}^{*} & \mu_{4 H_{1}}^{*} & \mu_{4 H_{2}}^{*} \\
\mu_{5 H_{0}}^{*} & \mu_{5 H_{1}}^{*} & \mu_{5 H_{2}}^{*}
\end{array}\right]
$$

Among them, $P_{j}=(j=1,2,3)$

\section{Experimental Results and Analysis}

In the experiment, the weight vector of the sensor is $(0.28,0.31,0.16,0.15,0.10)$.

In the fusion system, the results are:

Table 1 Experimental data

\begin{tabular}{cccc}
\hline Sensor & small & moderate & large \\
\hline Sensor 1 & 0.13 & 0.69 & 0.18 \\
Sensor 2 & 0.15 & 0.76 & 0.09 \\
Sensor 3 & 0.49 & 0.48 & 0.03 \\
Sensor 4 & 0.23 & 0.34 & 0.43 \\
Sensor 5 & 0.10 & 0.73 & 0.17 \\
\hline
\end{tabular}


According to the weighted average $M(\bullet,+)$, the results of the synthesis is

$$
\begin{aligned}
& P_{1}=\left(\begin{array}{lllll}
0.28 & 0.31 & 0.16 & 0.15 & 0.1
\end{array}\right)\left(\begin{array}{c}
0.13 \\
0.15 \\
0.49 \\
0.23 \\
0.1
\end{array}\right)=0.17 \\
& P_{2}=\left(\begin{array}{lllll}
0.28 & 0.31 & 0.16 & 0.15 & 0.1
\end{array}\right)\left(\begin{array}{l}
0.69 \\
0.76 \\
0.48 \\
0.34 \\
0.73
\end{array}\right)=0.63 \\
& P_{3}=\left(\begin{array}{lllll}
0.28 & 0.31 & 0.16 & 0.15 & 0.1
\end{array}\right)\left(\begin{array}{l}
0.18 \\
0.09 \\
0.03 \\
0.43 \\
0.17
\end{array}\right)=0.17 \\
& P=(0.17,0.63,0.17)
\end{aligned}
$$

The table 2 is a moment sensor respective membership value and its decision in accordance with the rules of warp tension and fusion are given after the results.

Table 2 Results of sensor alone recognition and fusion recognition

\begin{tabular}{cccccc}
\hline \multirow{2}{*}{ state } & Sensor i & \multicolumn{3}{c}{ Degree of membership } & \multirow{2}{*}{$\begin{array}{l}\text { detection } \\
\text { result }\end{array}$} \\
\cline { 2 - 4 } moderate & small & moderate & large & \\
& Sensor 1 & 0.13 & 0.69 & 0.18 & moderate \\
& Sensor 2 & 0.15 & 0.76 & 0.09 & moderate \\
& Sensor 3 & 0.49 & 0.48 & 0.03 & unknown \\
& Sensor 4 & 0.23 & 0.34 & 0.43 & unknown \\
& Sensor 5 & 0.10 & 0.73 & 0.17 & moderate \\
& result & 0.17 & 0.63 & 0.17 & moderate \\
large & Sensor 1 & 0.05 & 0.21 & 0.74 & large \\
& Sensor 2 & 0.08 & 0.25 & 0.66 & large \\
& Sensor 3 & 0.10 & 0.14 & 0.76 & large \\
& Sensor 4 & 0.09 & 0.45 & 0.46 & unknown \\
& Sensor 5 & 0.12 & 0.16 & 0.72 & large \\
& result & 0.10 & 0.24 & 0.68 & large \\
small & Sensor 1 & 0.69 & 0.24 & 0.07 & small \\
& Sensor 2 & 0.78 & 0.09 & 0.13 & small \\
& Sensor 3 & 0.49 & 0.42 & 0.09 & unknown \\
& Sensor 4 & 0.61 & 0.38 & 0.01 & small \\
& Sensor 5 & 0.78 & 0.17 & 0.05 & small \\
& result & 0.68 & 0.24 & 0.12 & small \\
\hline
\end{tabular}

It can be seen from the table, at a certain moment, when $P=(0.09,0.24,0.68)$, the maximum membership method is adopted for the results, it can be seen the warp tension in the large state. 


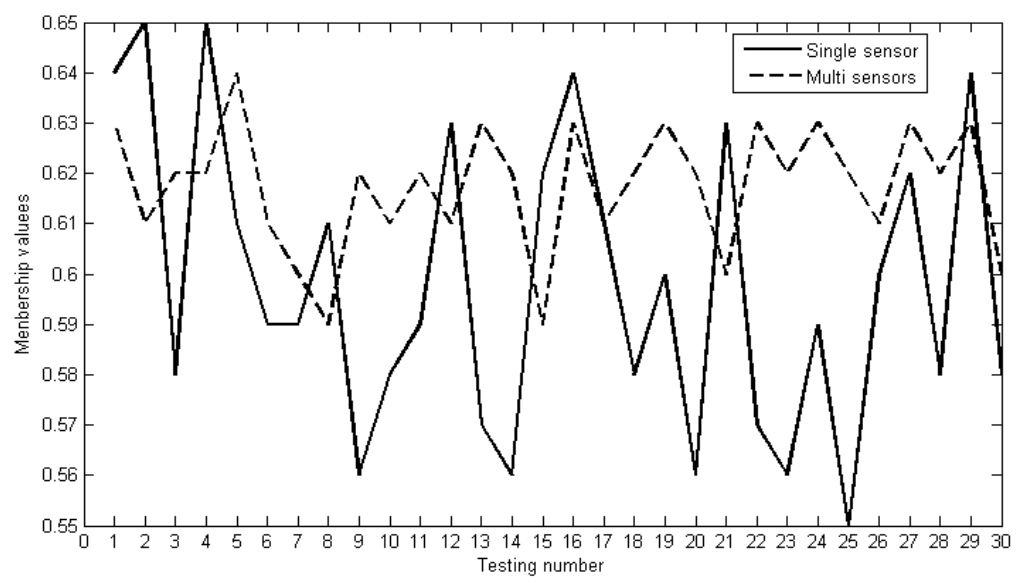

Fig.3 Curve of measured and fusion value

In the experiment, 30 group of warp tension testing data of sensor 1 membership value and fusion results were compared in figure 5 . Accuracy increased by $2.3 \%$. The results indicated that the fusion system uncertainty decreased significantly.

\section{Summary}

In the system of carbon fiber multi-layer loom, it have a great impact to three dimensional fabric weaving produced, as a result of warp tension control accuracy is not high. To improve the control precision of the system of warp tension and reduce the uncertainty of tension sensors, the neural network of the fuzzy comprehensive evaluation method is applied to send the system, make full use of the redundancy and complementarily of multi-source information. Experimental results show that the method enhances the warp tension testing reliability, and improves the control accuracy of the warp tension, it provides a theoretical basis for improving the control precision of the warp tension.

\section{References}

[1]. WU Zhenyu, CHEN Linrong, LI Zijun, et al. Dynamic measurement model of contact tension sensor for yarn[J].Journal of Textile Research, 2013,34(8) , p. 138-142.

[2]. NI Min-na,LI Ping, LI Yan.Schemes Selection of Warp Tension Measurement and Control Based on Fuzzy Multiple-Attribute Group Decision Making[J].Journal of DongHua University (Natural Science). 2014,40(3) , p. 282-287.

[3]. YANG Jiancheng, JIANG Xiuming, ZHOU Guoqing, et al. WU Dongfeng. Application of Fuzzy-PID compound control on loom electronic let-off and take-up[J]. Journal of Textile Research, 2008,29(4) , p. 115-118.

[4]. ZHOU Xiangqin, LIU Yisheng.Warp tension change caused by shedding on loom[J]. Journal of Textile Research, 2014,35(5) , p. 132-136.

[5]. DENG Hailong. Study on the control mode of electronic feeding mechanism in modern loom[J]. Textile Machinery. 2012 (03), p. 27-30.

[6]. FU Jiacai, WAN Sui. Multi sensor information fusion based on D-S evidence theory and BP neural network[J].Automation and instrumentation.2011(1) , p. 22-24.

[7]. Chaotic optimization algorithm for multi-objective 0-1 programming problem[J] . Application Research of Computers. 2012,29(12) , p. 4486-4488.

[8]. FU Hua, GAO Ting, LIU Yang. Application of multi-sensor fuzzy information fusion in mine safe-ty[J].Transducer and Microsystem Technologies. 2008,27 (5) , p. 114-120. 